\title{
Approaches, Issues and Challenges in Recommender Systems: A Systematic Review
}

\author{
Balraj Kumar $^{1 *}$ and Neeraj Sharma ${ }^{2}$ \\ 'School of Computer Applications, Lovely Professional University, Phagwara - 144411, Punjab, India; \\ balraj_kr@yahoo.co.in \\ 2Department of Computer Science, Punjabi University, Patiala - 147002, Punjab, \\ India; sharma_neeraj@hotmail.com
}

\begin{abstract}
Objectives: Today the recommendation technology has managed to achieve a distinct place in the modern and fascinating world of e-commerce applications as it helps the user in selecting items or products of his interest from a large pool. The present article aims to provide a comprehensive and systematic review of the state-of-the-art recommender systems. Methods/Statistical Analysis: The entire literature review process was divided into six research questions keeping in view the different perspectives of recommendation field. The methodology adopted here, consists of the search plan and the paper selection criteria. The search plan attempts to retrieve the research studies through several digital libraries and the paper selection criteria help filter out the most relevant studies further to gather evidence against each of the research questions. Findings: The literature review process provides a thorough discussion on different techniques deployed in recommender system literature such as collaborative filtering, content-based filtering, social filtering, demographic and knowledge-based and utility based systems. It also explores their strengths and weaknesses. The recommender systems face certain challenges in their deployment such as cold-start, sparsity, scalability, user privacy, etc. The different application domains where recommender systems are being adopted these days include movie, music, books, news, tourism etc. The gap analysis conducted during literature review, focuses on improving the traditional recommendation approaches, the precise blend of existing approaches with different types of information, modeling of user profiles and recommended items, standardization of non-standard evaluation techniques etc. Application/Improvements: This paper also throws some light on certain application fields such as television, research grants, restaurant, job search, etc. that need to grab the attention of scientific and research communities to promote more research in those areas.
\end{abstract}

Keywords: Application Domains, Collaborative Filtering, Evaluation Measures, Recommendation Approaches, Recommender Systems

\section{Introduction}

In today's Information Age, information overload is a major point of concern. The information overload actually indicates the availability of too much data or information that is beyond the manageable limits of the user and causes a big difficulty in all sorts of decision makings. This problem occurs mainly when the system is unable to handle and process this huge amount of information in a systematic manner. For example, in many e-commerce web applications, generally the user is presented with a plenty of options, but with a very limited time to explore them all. A recommendation system, the most powerful mechanism in this direction, attempts to tackle the information overload problem ${ }^{1}$. They are the common tools successfully deployed in many commercial environments such as Amazon, Netflix, Tripadvisor, Last.fm, etc.

${ }^{*}$ Author for correspondence 
A Recommender System (RS) may be defined as a set of software tools and techniques that provides commendations to the users for certain items that may be useful to them 2 . These suggestions help users in their routine decision-making. For instance, what movie to watch, what songs to listen to, what book to buy and what news to read, etc. A RS is usually visualized as a knowledge discovery tool as it has the ability to build new and diversified recommendations to meet the requirements of its users. A recommender system offers its services mainly to two kinds of players involved in e-commerce; the one is the service provider who aims to maximize his profits; the other is the user who actually enjoys its benefits in quality decision making with the availability of plethora of useful information based on the experience or preferences of others ${ }^{3}$.

Since the inception of recommendation field in the mid-1990s, a huge amount of literature has been published in this area. ${ }^{4}$ attempts to familiarize with the area of RSs; the recommendation techniques typically categorized into 3 major classes: content based, collaborative, and hybrid recommendations; and evaluation techniques along with their merits and limitations. ${ }^{5}$ intends to provide a comprehensive survey of collaborative filtering methods from the basic to advanced techniques, their tasks and major challenges with possible solutions. ${ }^{6}$ also provides a meticulous and thorough literature review of collaborative filtering methods and implicit feedback from user behavior and actions to gather evidence as a fusion of the scientific articles of best quality.

$\operatorname{In}^{7}$ provides a detailed review on recommender systems, their classification, application arenas and different data mining methods. ${ }^{-}$presents the classification framework where they classified the literature into 8 categories of RSs domains and 8 categories into data mining methods. Their findings shown that the movie field has received the maximum research, since this is the only domain that has been able to attract the research community the most. ${ }^{9}$ investigates the latest advances in RSs and talk over their key challenges. They also present the working and comparative study of existing algorithms and investigate their impacts in prospective developments.

This article intends to provide a detailed literature review in the RSs field. For this purpose, the entire review process has been divided into six research questions. These questions have been designed very carefully keeping in view the different perspectives of RSs area. The purpose of this study in broader sense is twofold: First, to provide an overview of the state-of-the-art approaches and methods used in different recommender application domains. Second, to present the trends and future directions to prospective researchers/practitioners who are looking for new research opportunities and are keen to pursue their research in the area of RSs.

The remainder of this paper is structured as follows: Section-2 introduces the research methodology adopted to carry out the present study. In Section-3, the review process identifies six research questions and their possible explanations to cover the different and broad perspectives of RSs. This section also highlights many research directions likely to be the focus of RSs research in near future. Section-4 describes the limitations of the present study. At last, Section-5 presents the final concluding remarks.

\section{Methodology}

This section presents the research methodology in the form of a flowchart as shown in Figure 1. It is based on 10,11 and has been described in terms of the search plan and the paper selection criteria. The present study aims to provide an understanding and insights of the recent trends in RS research by investigating the published literature. This methodology provides the basic steps to identify, understand and analyze the research articles and this in turn, facilitates in gathering evidence for answering the research questions (RQ1 to RQ6) (see Section 3). The following subsections describe the search plan and the criteria for selecting the papers:

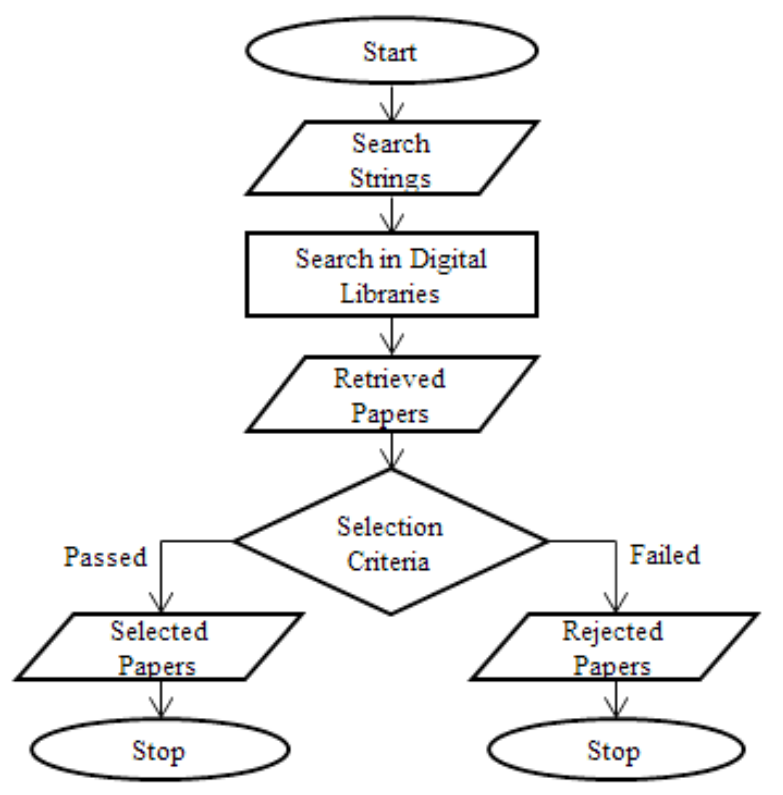

Figure 1. Relevant paper selection methodology. 


\subsection{Search Plan}

Under the search plan, a proper expert planning and validation of search strings was carried out. To find the relevant research publications, automatic search was carried through several digital libraries as given in Table 1. These libraries were selected as they are considered the most appropriate, very popular and rich source of research publications relevant to the field of recommendation systems.

The search strings were designed carefully based on the research questions. To compile almost all of the relevant publications, the search strings were repeatedly refined. Thus, various search strings with different combinations of terms were used while searching the relevant studies. These strings are: "recommender system" OR "recommendation system" OR "recommendation approaches" OR "recommendation methods" OR "recommender application domains"

According to these search strings, an automatic search was carried out through search engines of several digital libraries and a total of 290 studies were retrieved. Then, to filter out the most relevant studies further, the paper selection criteria were applied on them.

\subsection{Paper Selection Criteria}

After the search plan, the next step was to describe the paper selection criteria to choose the most relevant studies. The key selection criterion (the subjective one) for selecting a paper was its relevance/connection with the research questions. Other two types (the objective ones) are inclusion and exclusion criteria given in Table 2were decided for selecting the relevant basic studies ${ }^{10}$.

After the selection process, all the papers were segregated into two sets i.e. selected papers and rejected papers. Out of 290 studies, only 66 papers $(22.76 \%)$ were identified as relevant to the review study and the remaining 224 papers (77.24\%) were excluded. Table 3 presents the summarized results of the searches and the sources on which they were performed.

Table 1. The selected digital libraries

\begin{tabular}{|l|l|}
\hline Digital Library & URL \\
\hline SpringerLink & http://link.springer.com \\
\hline IEEE Xplore & http://ieeexplore.ieee.org \\
\hline ProQuest Database & http://search.proquest.com \\
\hline GoogleScholar & http://scholar.google.co.in \\
\hline ACM Digital Library & http://dl.acm.org \\
\hline
\end{tabular}

Table 2. Inclusion/exclusion criteria

\begin{tabular}{|c|}
\hline Type-I: Inclusion Criteria \\
\hline $\begin{array}{l}\text { Papers related to research questions (RQ1 to RQ6) } \\
\text { Full-papers } \\
\text { Peer-reviewed articles } \\
\text { Papers published between } 2001 \text { and 2016June } \\
\text { Papers written in English language only } \\
\end{array}$ \\
\hline Type-II: Exclusion Criteria \\
\hline $\begin{array}{l}\text { Papers not related to research questions (RQ1 to RQ6) } \\
\text { Short-papers and posters } \\
\text { Non peer-reviewed/unpublished working articles } \\
\text { Papers published before } 2001 \text { or after } 2016 \text { June } \\
\text { Papers written in Non-English languages } \\
\text { Duplicate papers } \\
\text { Master's and Doctoral dissertations }\end{array}$ \\
\hline
\end{tabular}

Table 3. Summary of search results

\begin{tabular}{|l|c|c|c|}
\hline Digital Library & $\begin{array}{c}\text { Search } \\
\text { Results }\end{array}$ & $\begin{array}{c}\text { Relevant } \\
\text { Studies }\end{array}$ & $\begin{array}{c}\text { \% of Total } \\
\text { Relevant } \\
\text { Studies }\end{array}$ \\
\hline SpringerLink & 66 & 18 & 27.27 \\
\hline IEEE Xplore & 62 & 11 & 16.67 \\
\hline ProQuest Computing & 72 & 13 & 19.70 \\
\hline GoogleScholar & 55 & 16 & 24.24 \\
\hline ACM Digital Library & 35 & 9 & 13.64 \\
\hline Total & 290 & 66 & 100 \\
\hline
\end{tabular}

\section{Research Questions in Focus}

This section describes six Research Questions (RQs) presented in Table 4, which are raised to provide a detailed review of various broader aspects of recommender systems. The following subsections attempt to present these aspects while answering the RQs:

\subsection{Recommendation Approaches}

The task of commendation can be viewed as the prediction of rating of an unrated item. Generally, for all those items where no ratings are given by a user, the rating predictions are computed and the top items with the highest ratings are presented as the recommendations ${ }^{12}$. The first research question is raised to gather evidence about the various RSs approaches. RQ1: What kinds of approaches are utilized for generating recommendations by RSs? The widely used recommendation technologies in commercial applications can be broadly classified as ${ }^{\frac{13}{3}}$ : 
Table 4. Research questions

\begin{tabular}{|l|l|}
\hline RQ\# & Research Question Statement \\
\hline RQ1 & $\begin{array}{l}\text { What kinds of approaches are used for generating } \\
\text { recommendations by RSs? }\end{array}$ \\
\hline RQ2 & $\begin{array}{l}\text { What are the strengths and weaknessesof } \\
\text { recommendation approaches practiced in RSs field? }\end{array}$ \\
\hline RQ3 & $\begin{array}{l}\text { What kinds of issues and challenges encounter in } \\
\text { deployment of RSs? }\end{array}$ \\
\hline RQ4 & $\begin{array}{l}\text { What are the various application domains where RSs } \\
\text { being adopted? }\end{array}$ \\
\hline RQ5 & $\begin{array}{l}\text { Which evaluation methods are used to measure the } \\
\text { quality of RSs? }\end{array}$ \\
\hline RQ6 & $\begin{array}{l}\text { What are the different gaps exist in the present RSs } \\
\text { research? }\end{array}$ \\
\hline
\end{tabular}

Collaborative filtering (CF) technique recommends the items to the target user on the basis of past preferences of other users with similar tastes ${ }^{\underline{14}}$. CF helps the users make choices based on the opinions of others. That's why it is also known as the word-of-mouth approach. Last.fm, Ringo and Video recommender are some of the examples of CF systems. CF techniques are further classified into two categories 1 : memory-based methods, that make the recommendations by exploiting the complete user database; and model-based methods, that first fit a model based on user database and then use it to make predictions. Content-based filtering (CB) recommends items which are similar to the ones a given user has liked in the past ${ }^{3}$. A profile is created for each user or item to describe its inherent characteristics. For instance, the attributes of a movie profile may be its genre, director, actors, its box office popularity etc. User profiles may include their selected items, ratings, demographic information etc. The profiles help recommender systems associate users with matching items. Pandora Internet Radio is the best example of this approach.

Social Filtering (SF), also known as communitybased RSs, recommend items based on the preferences of the users' friends. These RSs follow the saying "Tell me who your friends are and I will tell you who you are" 15 . Generally, users trust more on friends' recommendations than on by unknown people $e^{16}$. Such systems gather social information of users and preferences of their friends from social networks and generate the recommendations based on the ratings given by user's friends ${ }^{3}$. Demographic systems (DG) recommend items on the basis of user's demographic profile. These systems attempt to produce different recommendations for different demographic roles. For instance, several websites assume certain effective personalization mechanisms based on demographics like language, country or age $\mathrm{e}^{\mathrm{17}}$.

Knowledge-based systems (KB) rely on specific domain knowledge to recommend items to their users. They map the item features with user requirements and preferences to determine whether the item is useful for the user. Such systems are called case-based systems; where a similarity function evaluates the matching degree of user needs with the suggested recommendations $s^{18}$. Utility Based systems $(\mathrm{UB})^{2}$, like $\mathrm{KB}$ systems, base their recommendation on the matching assessment between the user needs and the choices available. These systems compute the utility of each item before recommending it to the users.

Hybrid RSs follow a blended approach that covers all other basic approaches to achieve some synergy among them ${ }^{3}$. Such a system that combines two techniques, attempts to use the advantages of one to fix the disadvantages of other ${ }^{19}$. For example, $\mathrm{CF}$ and $\mathrm{CB}$ approaches can be used together to avoid the new-item problems of $\mathrm{CF}$ techniques. In literature, there are many different ways proposed to create a hybrid system by merging two or more basic approaches ${ }^{20}$.

Apart from the above mentioned, several other approaches were also found in literature that include data mining techniques, used to extract knowledge from data and can be used to improve RSs performance; context aware techniques provide contextual information to provide proactive recommendations to its user without getting any explicit request; and semantic-based techniques use semantic similarity to determine the semantic relations between users' likings and items presented in domain ontology ${ }^{10}$. From the literature, it was learnt that $\mathrm{CF}$ and $\mathrm{CB}$ are the most popular approaches that have got the wide acceptance and extensive usage in RSs field over others.

\subsection{Strengths and Weaknesses of RSs Approaches}

In this subsection, a lot of scientific and technical literature has been reviewed to study and analyze the pros and cons of recommendation approaches. Each of these has its own strengths and weaknesses 2 , which are summarized in Table 5 and queried in the forthcoming research question. RQ2: What are the strengths and weaknesses of recommendation approaches practiced in RSs field? 
Table 5. Strengths and weaknesses of recommendation approaches

\begin{tabular}{|c|c|c|c|c|c|c|c|}
\hline \multicolumn{2}{|c|}{ Key Points } & $\mathrm{CF}$ & $\mathrm{CB}$ & DG & SF & $\mathrm{KB}$ & UB \\
\hline \multirow{11}{*}{ 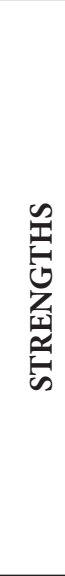 } & Identify cross-genre niches & $\sqrt{ }$ & & $\sqrt{ }$ & & & \\
\hline & No need of domain knowledge & $\sqrt{ }$ & $\sqrt{ }$ & $\sqrt{ }$ & & & \\
\hline & Adaptive nature as quality can improve over time & $\sqrt{ }$ & $\sqrt{ }$ & $\sqrt{ }$ & & & \\
\hline & Adequacy of implicit feedback & $\sqrt{ }$ & $\sqrt{ }$ & & & & \\
\hline & Free from cold-start user issue & & & & $\sqrt{ }$ & $\sqrt{ }$ & $\sqrt{ }$ \\
\hline & Free from cold-start item issue & & $\sqrt{ }$ & & & $\sqrt{ }$ & $\sqrt{ }$ \\
\hline & Sensitive to preference changes & & & & & $\sqrt{ }$ & $\sqrt{ }$ \\
\hline & Inclusion of non-product features & & & & & $\sqrt{ }$ & $\sqrt{ }$ \\
\hline & Mapping of user needs to product items & & & & & $\sqrt{ }$ & \\
\hline & Transparency in recommendations & & & & $\sqrt{ }$ & & \\
\hline & Trust, Scrutability and persuasiveness & & & & $\sqrt{ }$ & & \\
\hline \multirow{9}{*}{ 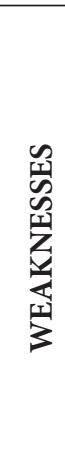 } & New user problem & $\sqrt{ }$ & $\sqrt{ }$ & $\sqrt{ }$ & & & \\
\hline & New item problem & $\sqrt{ }$ & & & & & \\
\hline & Problem of gray sheep & $\sqrt{ }$ & & $\sqrt{ }$ & & & \\
\hline & Quality depends on huge historical data-set & $\sqrt{ }$ & $\sqrt{ }$ & $\sqrt{ }$ & $\sqrt{ }$ & & \\
\hline & Stability Vs plasticity problem & $\sqrt{ }$ & $\sqrt{ }$ & $\sqrt{ }$ & & & \\
\hline & Gathering of demographic info is must & & & $\sqrt{ }$ & & & \\
\hline & Input of utility function by the user & & & & & & $\sqrt{ }$ \\
\hline & Static suggestion ability & & & & & $\sqrt{ }$ & $\sqrt{ }$ \\
\hline & Need of knowledge engineering & & & & & $\sqrt{ }$ & \\
\hline
\end{tabular}

CF recommends items based on taste similarity of other users. But it behaves very poorly for gray sheep that fall on a boundary among several user groups. Gray sheep are the users whose preferences do not consistently agree/ disagree with any user group and thus do not benefit from CF techniques ${ }^{5}$. Other challenges of CF techniques include dealing with data sparsity, scalability, cold-start issues etc. (see Section 3.3). CB methods also face coldstart user problem as they need to gather adequate ratings to generate quality recommendations. $\mathrm{CF}$ and $\mathrm{CB}$ both are of adaptive nature and can build recommendations with implicit feedback and no domain knowledge.

DG Systems (like CF) also face the problem of gray sheep because here the users are categorized on personal characteristics. But these systems are free from any new user problem because they don't need any list of user ratings. Rather there is a challenge of collecting the necessary demographic information. SF systems provide recommendations based on user's profiles and their social/trust network. Their plus points are transparency, alleviation of sparsity and cold-start user issues whereas the lack of suitable and publically available test data sets is the major shortcoming.

$K B$ and $U B$ recommenders don't have any data sparsity or cold start issues. KB systems are the best for casual exploration since they need less user details than UB systems, and they can make wide-range recommendations depending upon their knowledge-base, but they cannot determine user roles, the way CF systems can do. KB systems suffer from some other problems associated with the acquisition of user knowledge, catalog knowledge and features of items, and functional knowledge for mapping between user needs and the items. UB systems create a utility function to compute the utility of an item prior to its recommendation to a user. Their key benefit is that several factors such as delivery schedule, warranty terms etc. not only add product-specific features, but also may contribute to the product value.

The learning-based approaches such as CF, CB and DG suffer from stability vs. plasticity issue. If user's 
profile is already there in the system, then there is a challenge in changing its's preferences. For example, if a non-vegetarian turns into a vegetarian after some period, he may continue to get recommendations for non-veg items from a CF or $\mathrm{CB}$ recommenders for some-time, until the new ratings got the chance to take over ${ }^{2}$.

\subsection{Issues and Challenges in RSs}

This subsection describes the most common issues and challenges that encounter in deploying RSs and are considered important in the RSs research ${ }^{3}$. This perspective is raised in the next research question, i.e. RQ3: What kinds of issues and challenges encounter in deployment of RSs?

Sparse RSs: Generally, majority of the users do not rate most of the items and consequently the ratings matrix becomes very sparse. Due to this, the data sparsity problem arises that declines the chances of finding a set of users with similar ratings ${ }^{21,22}$. This is the most eminent drawback of the CF technique. This concern can be alleviated by using some additional domain information $\underline{23}$.

Cold-start problem: Cold-start problem presents a collective issue of new item and new user to RSs ${ }^{24}$. A new item can't be recommended initially when it is introduced to a CF system with no ratings. For instance, MovieLens (movielens.org) cannot recommend new movies until these have got some initial ratings. The new-user problem is bit hard to handle because it is not possible to find similar users or to create a $\mathrm{CB}$ profile without previous preferences of a user ${ }^{12}$.

Scalability Problem: One vital and foremost issue of RSs today is the scalability of algorithms with large real-world datasets. It is becoming challenging to deal with huge and dynamic data sets produced by item-users interactions such as preferences, ratings and reviews. It is possible that when some recommendation algorithms are applied on relatively small data sets, they provide the best results, but may reflect inefficient or worst behavior on very large datasets. Thus, some advanced large-scale assessment methods are required to deal with this issue $\mathrm{e}^{25,26}$.

Privacy Issue: To produce quality personalized recommendations, RSs are bound to gather as much user data as possible and to exploit it to the fullest. But on the other side, this may create a negative impression on the users' mind about their privacy because the system knows too much about them. Thus, such techniques need to be designed that can sensibly, meticulously and carefully use the user data by ensuring that information about the users' true preferences is not freely accessible to malevolent users ${ }^{27}$.

Robustness of RSs: Another major challenge in RSs is its robustness to attacks. Robustness is a performance measure of RSs. To gain certain profits, an attacker may generate some fake user profiles based on some attack models, such as Push/Nuke Attacks to make some target items more/less popular respectively. Such attacks are collectively called shilling attacks or profile injection attacks ${ }^{28}$.

Recommenders in Mobile Devices: Locationbased services are becoming more popular these days with the swift development of wireless networks and mobile devices; hence the geographical information is having a vital role to play here ${ }^{29}$. The users may seek different recommendations especially when they are to move across cities, hotels, restaurants, shops etc. Such type of scenario demands for the possible computational solutions along with mobile user interfaces that can effectively and efficiently utilize the available limited resources such as the screen size and computing power of the mobile devices.

Proactive RSs: There are RSs that are responsible for making recommendations even if not explicitly requested ${ }^{30}$. Mostly the RSs developed so far follow a "pull model" where the recommendation requests are initiated by the users ${ }^{31}$. In today's modern life, where users are always connected to world of computing and internet, it is desirable to have such systems that can predict what, when and how to "push" recommendations on implicit requests. Thus, the recommender systems can reflect proactive approach while recommending items in the interest of user ${ }^{3}$.

Diversity of Items: Generally, a user can opt for an item of his interest from a recommendation list if the list reflects some diversity in the recommended items to some extent. Seamless recommendations for a restricted type of product have no value until or unless it is desired or explicitly described by the user with a narrow clique of preferences. In the initial stage, when the RS is used as a knowledge discovery tool, the users may wish to explore new and different options available. Till date not much research has been carried out on this topic. But the need of hour is to design such solutions that could achieve the goal of diversity of items together with the accuracy of recommendation ${ }^{32}$. 


\subsection{Recommender Application Domains}

A recommendation domain refers to the set of items a recommender works upon to achieve some specified objective. From the literature review, it is learned that RSs have been designed and developed to recommend a variety of items that can broadly be classified into several domains of interest. These items include books, webpages, news, research articles, documents, restaurants, music, movies, jokes, etc. Here ${ }^{3,33}$ the perspective of items and their associated domains arises from the following research question: RQ4: What are the various application domains where RSs being adopted?

Web Recommenders: Now-a-daysRSs are increasingly being adopted in websites where they play a significant role. The review findings help identify many popular web recommenders. To learn about the users' personal interests, Personal WebWatcher keeps track of the Web pages they visit and also the pages laying just a link away from the visited ones. if Web uses advanced representation techniques to represent profiles in the form of a weighted semantic network. It gathers both user's likings as well as dislikings from explicit feedback ${ }^{34}$.

E-Commerce: Recommender systems embedded in commercial applications provide recommendations to the customers and boost them to buy different kinds of products of their interest such as watches, cameras, PCs, books, etc. The product recommendations depend on demographic information, or past purchasing behavior of the customer. RSs enhance e-commerce sales by converting browsers into buyers, increasing cross-sell by recommending supplementary products and gaining customer loyalty. Examples of commercial RSs include amazon, eBay, drugstore, moviefinder, reel, cdnow ${ }^{31}$.

Movie: There has been a significant increase in the movie RSs since it attracts almost all categories of users and researchers. MovieLens $s^{35}$ recommends movies to the users based on their movie preferences. Netflix $x^{36}$ is also a recommender for movies and TV shows that allows streaming on internet-connected devices at any time. Movies $2 \mathrm{GO}^{37}$ an online voting based movie RS, learns user preferences from movie synopsis rated by the user. On the same lines, television RSs assist TV watchers by recommending interesting TV programmes to watch more comfortably and avoiding the traditional way of channel surfing ${ }^{38}$.

Music: In the music domain ${ }^{39}$, $\mathrm{CF}$ techniques are very common in producing recommendations. Last.fm and
MyStrands RSs are the best examples. Pandora is another popular music recommender that uses $\mathrm{CB}$ filtering technique. FOAFing the music ${ }^{40,41}$ is another recommender that discovers, explores and recommends the music content based on user profiling via Friend of a Friend ( $w w w$. foaf-project.org) description, content-based and contextbased information.

News: This category provides very exciting recommendations about a variety of breaking news to its potential consumers. YourNews ${ }^{42}$ is a RS for personalized news access where a distinct interest profile is maintained for various topics such as Business National, World, etc. It can recommend recent news using profiles. Daily Learner ${ }^{39,43}$ is a learning agent for wireless information access that learns two separate short-term and long-term user-models.

Tourism: ${ }^{44}$ Tourism recommenders produce personalized recommendations for the tourists whenever they are to visit unfamiliar places. These recommenders serve them as support tools to make their decision process simple, easier and more manageable. Tourism RSs employ two types of interfaces: web-based which are very useful prior to the visit and mobile-based that recommends attractions during the visit. Examples of web-based RSs include e-Tourism ${ }^{45}$, City Trip Planner ${ }^{46}$ and Otium ${ }^{47}$; whereas MapMobyRek ${ }^{48}$, GeOasis ${ }^{49}$ (a tourist guide that uses GPS) and Android based RSs like moreTourism ${ }^{50}$ and $S T S^{51}$ are the examples of mobile-based RSs.

Document/Information: Several RSs have been proposed in literature that belongs to this category. These are the applications that provide the most valuable information or documents to a large user community such as scientific and research community etc. on a single click. Commonly used RSs are described as follows: CYCLADES ${ }^{52}$ recommends documents to users considered as relevant just like alerting services. It provides a common platform where information can be searched, shared and organized as per user's own view. In DocCloud ${ }^{53}$, a service provider offers a document as a service using the SaaS paradigm of cloud computing. Quickste $p^{54}$ provides recommendations of online available academic research articles and documents.

Miscellaneous: In this category, all other recommendation fields can be put together wherein till date the research is very limited. These domains include recommendation of learning resources, research grants, IT skills, images, jokes, real estate, restaurant, job search, similar user recommendation on $\mathrm{P} 2 \mathrm{P}$ network, etc ${ }^{33}$. 
Few examples include CoFIND ${ }^{55}$, FlatFinder ${ }^{\frac{56}{6}}$, CASPER $^{57}$, PolyLens ${ }^{58}$, Eigentaste ${ }^{\frac{59}{}}$.

Thus, RSs are being deployed in diverse application areas like movies, music, news, webpages, books, restaurants, and many other products. Also, to satisfy user's information needs, the RSs can even recommend the potential queries to search information based on user's search history ${ }^{60}$. Here, the findings have revealed that there are several RSs that charge for the services offered, while others recommend items to users for free of cost.

\subsection{Evaluation Measures of RSs}

The quality of recommender systems is judged on the basis of result of evaluation. Since the inception of recommenders, the assessment of predictions and recommendations has been considered important so that the users can have the best experience with RSs. The RSs research needs different metrics or measures to judge the quality of various methods, techniques and algorithms used for producing recommendations ${ }^{61}$. The evaluation point of view of RSs is highlighted from the following research question: RQ5: Which evaluation methods are used to measure the quality of $R S s$ ?

The evaluation measures may or may not operate with the recommendation algorithms employed in RSs. Also the objectives of assessment may be different in different scenarios ${ }^{62}$. Thus, the evaluation of RSs and their algorithms is quiet challenging. There are ample numbers of evaluation methods available in literature defined with the goal of making better selections. The classification of these metrics is a very tedious job. But, this paper presents the categorization of these metrics based on the classification provided in ${ }^{10,63}$ as follows:

Probabilistic Metrics: The probabilistic measures are very effective when used in evaluating the reliability of predictions produced by recommenders irrespective of the recommendations of unrelated items with low or high probability. The key examples of these metrics include Mean Absolute Error (MAE), Root Mean Squared Error (RMSE), LogLoss or cross-entropy. When actual rating of an item is compared with the predicted rating of RS, the recommendation task is viewed as a regression problem.

Qualitative Metrics: Qualitative metrics are very common in the RSs field and are very useful when the aim is to have a model to reduce the number of errors. These measures are very widely used in various RS applications. The key instances include Accuracy, F-measure, Kappa statistic, Coverage, etc. Few of them are very suitable for balanced/imbalanced datasets, signal/fault detection or information retrieval. During quality assessment, items are classified as relevant or irrelevant for a user. Then some metric is chosen to assess the quality of the items recommended by the RSs. Here the problem of recommendation is viewed as a classification problem.

Ranking Metrics: These metrics, broadly used in RSs, are based on the idea of how well the recommender ranks the recommended items. The main examples of these measures include Precision, Recall, Normalized Discounted Cumulative Gain (NDCG), Mean Average Precision (MAP), Hit Rate (HR), Fallout, Area under the ROC Curve (AUC), and so on. In comparison to previous categories, the present metrics class evaluates the quality of a ranked list of items instead of the average quality of the raw scores produced by the recommender system. In this category, the commendation task is visualized as a ranking problem.

User Satisfaction Metrics: These are the metrics where empirical experiments are conducted with users to assess their satisfaction level in recommender systems. This measure is widely used in numerous recommender systems and collects personal users feedback. This metric may have certain issues such as biases, lack of an objective measure for RS quality assessment, comparison among diverse systems etc.

\subsection{Gaps in RSs Research and Future Directions}

The main focus of future research is mostly on advancing the existing approaches and algorithms to enhance the quality of recommendations $s^{20}$. In the light of review of the literature and state-of-the-art developments in RSs field, many research gaps have been identified which are thought-provoking and may be considered worth investigating further. The following research question is raised in order to gather evidence about the gaps that exist in the present recommendation field. RQ6: What are the different gaps exist in the present RSs research? The findings of this work deliver the prospective researchers or practitioners with the acumens and future directions on RSsthat are presented in Table 6 in the form of gap analysis.

From the gap analysis extracted from RSs literature, it can be concluded that the area of RSs provides ample opportunities to scientific and research communities wherein they can investigate its different directions based 
Table 6. Gap analysis

\begin{tabular}{|c|c|}
\hline Contribution to RSs & Research Gaps \\
\hline $\begin{array}{l}{ }^{20} \text { provides the insights of RSs, their evolution, CF techniques, } \\
\text { algorithms, evaluation metrics and use of social information for } \\
\text { generating recommendations }\end{array}$ & $\begin{array}{l}\text { The precise blend of existing approaches, incorporation of tastes } \\
\text { and habits trends in recommendation practice, security and } \\
\text { privacy issues and standardization of evaluation methods. }\end{array}$ \\
\hline $\begin{array}{l}\text { The study by }{ }^{64} \text { presents an overview of review-based RSs where } \\
\text { review elements are obtained through advanced text analysis } \\
\text { and opinion mining techniques. They are exploited to improve } \\
\text { CF, CB and choice-based techniques. }\end{array}$ & $\begin{array}{l}\text { In review-based RSs, different combinations of various review } \\
\text { elements may be explored for better accuracy of user/item } \\
\text { profile; the reviews could be used to augment the quality of } \\
\text { recommendation explanations and these RSs can be combined } \\
\text { with other RSs to build multi-criteria RSs. }\end{array}$ \\
\hline $\begin{array}{l}{ }^{65} \text { proposes a relational collaborative topic regression model for } \\
\text { RSs. It utilizes some auxiliary info (item relations) to alleviate } \\
\text { sparsity problem faced by traditional CF methods. }\end{array}$ & $\begin{array}{l}\text { The proposed model can be adapted for social networks among } \\
\text { users. Moreover, some distributed learning algorithms can be } \\
\text { designed to enhance the scalability of proposed model for large } \\
\text { datasets. }\end{array}$ \\
\hline $\begin{array}{l}{ }^{66} \text { describes the needs, issues and challenges in designing RSs for } \\
\text { e-learning environments along with the drawbacks of different } \\
\text { recommendation methods. }\end{array}$ & $\begin{array}{l}\text { The problems of Tag-based RSs in e-learning environments } \\
\text { include uncontrolled vocabulary and tag ambiguity, tag } \\
\text { redundancy, tags with little semantics but different variations. }\end{array}$ \\
\hline $\begin{array}{l}10 \text { provides the classification, synthesis and present different } \\
\text { studies according to several development perspectives of RSs in } \\
\text { TV domain that includes approaches, algorithms, architectural } \\
\text { models, etc. }\end{array}$ & $\begin{array}{l}\text { Further expolration of modelling of user profiles and } \\
\text { recommended items, datasets used in RSs training, impact of } \\
\text { distributed architectures on RSs approaches and algorithms, } \\
\text { impact of explanations on the quality of recommendations, }\end{array}$ \\
\hline $\begin{array}{l}\text { The study by }{ }^{5} \text { aims to provide a comprehensive survey of CF } \\
\text { methods from the basic to advanced techniques, their tasks and } \\
\text { major challenges with possible solutions. }\end{array}$ & $\begin{array}{l}\text { Need of such a recommendation approach that is easy to } \\
\text { implement with few resources, produces accurate predictions } \\
\text { and recommendations, and overcomes all types of issues and } \\
\text { challenges presented by real world RSs. }\end{array}$ \\
\hline
\end{tabular}

on their interest and need of hour. Various possible future advancements in RSs field are summarized as follows:

The new research directions aim for improving the traditional recommendation approaches; the precise blend of existing approaches with different types of information; Modeling of user profiles and recommended items, standardization of non-standard evaluation techniques. To alleviate the sparsity problem of CF systems, the social networks among users can be adapted to make more robust systems. Some distributed learning algorithms can be designed to enhance the scalability of systems to handle large datasets. Different combinations of review elements for better accuracy and different combinations of review-based RSs with other RSs to build multi-criteria RSs. Very limited research has been carried out on this.

Tag-based RSs in e-learning environments have many problems to deal with like the problems of uncontrolled vocabulary and tag ambiguity, tag redundancy, tags with little semantics but different variations, etc. Moreover, development of a finest approach that takes care of different types of issues, like data sparsity, cold-start, scalability, privacy protection, etc $\frac{5,67}{}$.

\section{Limitations of Present Study}

The present study is having certain limitations which are expressed as follows: (a) The results obtained while searching the digital libraries (IEEE, Springer, ProQuest, etc) are not exhaustive and hence restrict to the research conducted. (b) There is no uniform and standard way of performing the search across all the digital libraries. (c) The findings presented in this study are purely based on the papers published in English language only since all non-English publications were ignored. (d) The selection of studies was based on title of the study, abstract and keywords. In many of the cases, the articles were identified on the basis of their full text as well. (e) Author bias for paper selections can be another challenge to this study. 


\section{Conclusion}

The recommender systems research has evolved out of information retrieval and filtering, and its gradual development has turned it into a very robust and challenging field of research on its own. This paper provides a systematic review of the state-of-the-art RSs embedded in many application fields. In this paper, out of 290 retrieved studies from the scientific literature, only 66 papers relevant to the research questions were identified. Based on the extracted data, different perspectives of the RSs were studied and investigated. The findings of this paper have many significant implications: First, extensively reviewed literature has shown that the field of RSs is still widely accepted area of research by the prospective practitioners and researchers. It has massive potential of growth in future. Second, though there are many techniques available in the RSs field, but still CF and CB approaches have managed to get the wide acceptance and extensive usage by the research community over others. Third, there are certain application fields that need to grab the attention of scientific and research communities to promote more research in those areas. These fields include Music, Television etc. Fourth, many RS research studies apply different evaluation methods especially the ranking measures because there is a huge increase in the recommendation algorithms that can be assessed comfortably with these metrics.

\section{References}

1. Liu J, Jiang Y, Li Z, Zhang X, Lu H. Domain-sensitive recommendation with user-item subgroup analysis. IEEE Transactions on Knowledge and Data Engineering. 2016 Apr; 28(4):939-50.

2. Burke R. Hybrid recommender systems: Survey and experiments. User modeling and user-adapted interaction.2002 Nov, 12(4):331-370.

3. Ricci F, Rokach L, Shapira B. Introduction to recommender systems handbook. Springer US, 2011, pp. 1-35.

4. Adomavicius G, Tuzhilin A. Toward the next generation of recommender systems: A survey of the state-of-the-art and possible extensions. IEEE Transactions on Knowledge and Data Engineering, 2005 Jun; 17(6):734-49.

5. Su X, Khoshgoftaar TM. A survey of collaborative filtering techniques. Advances in artificial intelligence. New York; 2009 Jan. 4:1-99.

6. Najafabadi MK, Mahrin MN. A systematic literature review on the state of research and practice of collaborative filtering technique and implicit feedback. Artificial Intelligence Review. 2016 Feb; 45(2):167-201.

7. Park DH, Kim HK, Choi IY, Kim JK. A literature review and classification of recommender systems research. Expert Systems with Applications. 2012 Sep, 39(11):10059-72.

8. Park DH, Kim HK, Kim JK, Choi IY, Kim JK. A review and classification of recommender systems research. International Proceedings of Economics Development and Research. 2011; 5(1):290-4.

9. Lü L, Medo M, Yeung CH, Zhang YC, Zhang ZK, Zhou T. Recommender systems. Physics Reports. 2012 Oct; 519(1):1-49.

10. Véras D, Prota T, Bispo A, Prudêncio R, Ferraz C. A literature review of recommender systems in the television domain. Expert Systems with Applications. 2015 Dec; 42(22):9046-76.

11. Felderer M, Fourneret E. A systematic classification of security regression testing approaches. International Journal on Software Tools for Technology Transfer. 2015 Jun; 17(3):305-19.

12. Melville P, Sindhwani V. Recommender systems. Encyclopedia of machine learning. Springer US; 2011. p. 829-38.

13. Elahi M, Ricci F, Rubens N. A survey of active learning in collaborative filtering recommender systems. Computer Science Review; 2016 May.

14. Schafer JB, Frankowski D, Herlocker J, Sen S. Collaborative filtering recommender systems. The adaptive web. Springer Berlin Heidelberg; 2007. p. 291-324.

15. Arazy O, Kumar N, Shapira B. Improving social recommender systems. IT Professional Magazine. 2009 Jul; 11(4):31-7.

16. Sinha RR, Swearingen K. Comparing recommendations made by online systems and friends. DELOS workshop: Personalisation and Recommender Systems in Digital Libraries; 2001 Jun. p. 106.

17. Mahmood T, Ricci F. Towards learning user-adaptive state models in a conversational recommender system. LWA; 2007. p. 373-8.

18. Bridge D, Göker MH, McGinty L, Smyth B. Case-based recommender systems. The Knowledge Engineering Review. 2005 Sep; 20(03):315-20.

19. Sharma R, Singh R. Evolution of recommender systems from ancient times to modern era: A survey. Indian Journal of Science and Technology. 2016 May; 9(20):1-12.

20. Bobadilla J, Ortega F, Hernando A, Gutiérrez A. Recommender systems survey. Knowledge-Based Systems. 2013 Jul; 46:109-32.

21. Sharma SK, Suman U. An efficient semantic clustering of URLs for web page recommendation. International Journal of Data Analysis Techniques and Strategies. 2013 Jan; 5(4):339-58. 
22. Ma H, Yang H, Lyu MR, King I. Sorec: Social Recommendation using probabilistic matrix factorization. Proceedings of the $17^{\text {th }} \mathrm{ACM}$ Conference on Information and Knowledge Management; 2008 Oct. p. 931-40.

23. Melville P, Mooney RJ, Nagarajan R. Content-boosted collaborative filtering for improved recommendations. In Proceeding Eighteenth national conference on Artificial intelligence; 2002 Jul. p. 187-92.

24. Schein AI, Popescul A, Ungar LH, Pennock DM. Methods and metrics for cold-start recommendations. Proceedings of the $25^{\text {th }}$ Annual International ACM SIGIR Conference on Research and Development in Information Retrieval; 2002 Aug. p. 253-60.

25. Han P, Xie B, Yang F, Shen R. A scalable P2P recommender system based on distributed collaborative filtering. Expert Systems with Applications. 2004 Aug; 27(2):203-10.

26. George T, Merugu S. A scalable collaborative filtering framework based on co-clustering. Proceedings of $5^{\text {th }}$ IEEE International Conference on Data Mining. 2005 Nov; p. 625-8.

27. Shyong K, Frankowski D, Riedl J. Do you trust your recommendations? An exploration of security and privacy issues in recommender systems. Emerging Trends in Information and Communication Security. Springer Berlin Heidelberg; 2006. p. 14-29.

28. Aghili G, Shajari M, Khadivi S, Morid MA. Using genre interest of users to detect profile injection attacks in movie recommender systems. Proceedings of $10^{\text {th }} \mathrm{IEEE}$ International Conference on Machine Learning and Applications (ICMLA); 2011 Dec. p. 49-52.

29. Singh SP, Singh P. Design and implementation of a location-based multimedia mobile tourist guide system. International Journal of Information and Communication Technology. 2014 Dec; 7(1):40-51.

30. Sae-Ueng S, Pinyapong S, Ogino A, Kato T. Personalized shopping assistance service at ubiquitous shop space. Proceedings of $22^{\text {nd }}$ IEEE International Conference on Advanced Information Networking and ApplicationsWorkshops (AINAW); 2008 Mar. p. 838-43.

31. Schafer JB, Konstan JA, Riedl J. E-commerce recommendation applications. Applications of Data Mining to Electronic Commerce. Springer US; 2001. p. 115-53.

32. Hwang CS, Kuo N, Yu P. Representative-based diversity retrieval. Proceedings of 3rd IEEE International Conference on Innovative Computing Information and Control (ICICIC); 2008 Jun. p. 155.

33. Rao KN. Application domain and functional classification of recommender systems-a survey. DESIDOC Journal of Library and Information Technology. 2008 May; 28(3):17-35.

34. Montaner M, López B, De La Rosa JL. A taxonomy of recommender agents on the internet. Artificial Intelligence Review. 2003 Jun; 19(4):285-330.
35. Jelassi MN, Ben Yahia S, MephuNguifo E. A personalized recommender system based on users' information in folksonomies. Proceedings of $22^{\text {nd }}$ International Conference on World Wide Web Companion; 2013 May. p. 1215-24.

36. Gomez-Uribe CA, Hunt N. The netflix recommender system: algorithms, business value, and innovation. ACM Transactions on Management Information Systems (TMIS). 2016 Jan; 6(4).

37. Mukherjee R, Jonsdottir G, Sen S, Sarathi P. MOVIES2GO: An online voting based movie recommender system. Proceedings of $5^{\text {th }}$ ACM International Conference on Autonomous Agents; 2001 May. p. 114-15.

38. Kompatsiaris Y, Merialdo B, Lian S. TV content analysis: Techniques and applications. CRC Press; 2012 Mar.

39. Lops P, De Gemmis M, Semeraro G. Content-based recommender systems: State of the art and trends. Recommender Systems Handbook, Springer US; 2011. p. 73-105.

40. Celma Ò, Ramírez M, Herrera P. Foafing the music: A music recommendation system based on RSS feeds and user preferences. International Society of Music Information Retrieval; 2005.

41. Celma Ò, Serra X. FOAFing the Music: Bridging the semantic gap in music recommendation. Web Semantics: Science, Services and Agents on the World Wide Web. 2008; 6(4):250-6.

42. Ahn JW, Brusilovsky P, Grady J, He D, Syn SY. Open user profiles for adaptive news systems: help or harm? Proceedings of $16^{\text {th }}$ ACM International Conference on World Wide Web; 2007 May. p. 11-20.

43. Billsus D, Pazzani MJ, Chen J. A learning agent for wireless news access. Proceedings of $5^{\text {th }}$ ACM International Conference on Intelligent User Interfaces; 2002 Jan. p. 33-6.

44. Borràs $\mathrm{J}$, Moreno $\mathrm{A}$, Valls $\mathrm{A}$. Intelligent tourism recommender systems: A survey. Expert Systems with Applications. 2014 Nov; 41(16):7370-89.

45. Sebastia L, Garcia I, Onaindia E, Guzman C. e-Tourism: a tourist recommendation and planning application. International Journal on Artificial Intelligence Tools. 2009 Oct; 18(5):717-38.

46. Vansteenwegen P, Souffriau W, Berghe GV, Van Oudheusden D. The city trip planner: an expert system for tourists. Expert Systems with Applications. 2011 Jun; 38(6):6540-6.

47. Montejo-Ráez A, Perea-Ortega JM, García-Cumbreras MÁ, Martínez-Santiago F. Otiŭm: A web based planner for tourism and leisure. Expert Systems with Applications. 2011 Aug; 38(8):10085-93.

48. Ricci F, Nguyen QN, Averianova O. Exploiting a mapbased interface in conversational recommender systems for mobile travelers. Tourism Informatics: Visual Travel Recommender Systems, Social Communities, and User Interface Design: IGI Global, Information Science Reference; 2009 Sep. p. 73-93. 
49. Santiago FM, López FA, Montejo-Ráez A, López AU. GeOasis: A knowledge-based geo-referenced tourist assistant. Expert Systems with Applications. 2012 Oct; 39(14):11737-45.

50. Rey-López M, Barragáns-Martínez AB, Peleteiro A, Mikic-Fonte F, Burguillo JC. moreTourism: Mobile Recommendations for tourism. Proceedings of IEEE International Conference on Consumer Electronics (ICCE), Las Vegas (USA); 2011 Jan.

51. Braunhofer M, Elahi M, Ricci F, Schievenin T. Context-aware points of interest suggestion with dynamic weather data management. Information and Communication Technologies in Tourism. Springer International Publishing; 2014. p. 87-100.

52. Avancini H, Candela L, Straccia U. Recommenders in a personalized, collaborative digital library environment. Journal of Intelligent Information Systems. 2007 Jun; 28(3):253-83.

53. Vera-del-Campo J, Pegueroles J, Hernández-Serrano J, Soriano M. DocCloud: A document recommender system on cloud computing with plausible deniability. Information Sciences. 2014 Feb; 258:387-402.

54. Middleton SE, Shadbolt NR, De Roure DC. Ontological user profiling in recommender systems. ACM Transactions on Information Systems (TOIS). 2004 Jan; 22(1):54-88.

55. Dron J, Mitchell R, Siviter P, Boyne C. CoFIND - an experiment in N-dimensional collabo-rative filtering. Journal of Network and Computer Applications. 2000 Apr; 23(2):131-42.

56. Viappiani $\mathrm{P}, \mathrm{Pu} \mathrm{P}$, Faltings B. Conversational recommenders with adaptive suggestions. Proceedings of ACM Conference on Recommender Systems; 2007 Oct. p. 89-96.

57. Lee DH, Brusilovsky P. Fighting information overflow with personalized comprehensive information access: A proactive job recommender. Proceedings of $3^{\text {rd }}$ IEEE International Conference on Autonomic and Autonomous Systems (ICAS07); 2007 Jun. p. 21.
58. O'connor M, Cosley D, Konstan JA, Riedl J. PolyLens: A recommender system for groups of users. Proceedings of $7^{\text {th }}$ European Conference on Computer-Supported Cooperative Work, Springer Netherlands; 2001 Sep. p. 199-218.

59. Goldberg K, Roeder T, Gupta D, Perkins C. Eigentaste: A constant time collaborative filtering algorithm. Information Retrieval. 2001 Jul; 4(2):133-51.

60. Park K. The potential knowledge recommendation system using User's search logs. Indian Journal of Science and Technology. 2016 Jun; 9(24):1-7.

61. Gunawardana A, Shani G. A survey of accuracy evaluation metrics of recommendation tasks. The Journal of Machine Learning Research. 2009 Dec; 10:2935-62.

62. Herlocker JL, Konstan JA, Terveen LG, Riedl JT. Evaluating collaborative filtering recomm-ender systems. ACM Transactions on Information Systems (TOIS). 2004 Jan; 22(1):5-53.

63. Ferri C, Hernández-Orallo J, Modroiu R. An experimental comparison of performance measures for classification. Pattern Recognition Letters. 2009 Jan; 30(1):27-38.

64. Chen L, Chen G, Wang F. Recommender systems based on user reviews: The state of the art. User Modeling and UserAdapted Interaction. 2015 Jun; 25(2):99-154.

65. Wang H, Li WJ. Relational collaborative topic regression for recommender systems. IEEE Transactions on Knowledge and Data Engineering. 2015 May; 27(5):1343-55.

66. Klašnja-Milićević A, Ivanović $M$, Nanopoulos A. Recommender systems in e-learning environments: a survey of the state-of-the-art and possible extensions. Artificial Intelligence Review. 2015 Dec; 44(4):571-604.

67. Perugini S, Gonçalves MA, Fox EA. Recommender systems research: A connection-centric survey. Journal of Intelligent Information Systems. 2004 Sep; 23(2):107-43. 